\title{
Raman microspectroscopic characterization of amorphous silica plastic behavior
}

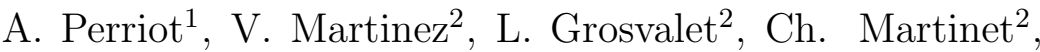 \\ B. Champagnon ${ }^{2}$, D. Vandembroucq ${ }^{1 *}$ and E. Barthel ${ }^{1 *}$ \\ 1 Surface du Verre et Interfaces, UMR 125 CNRS/Saint-Gobain, Aubervilliers, France. \\ ${ }^{2}$ Laboratoire de Physico-Chimie des Matériaux Luminescents, \\ UMR 5620 CNRS/Université Claude Bernard-Lyon I, Villeurbanne, France.
}

(Dated: August 16, 2016)

\begin{abstract}
Raman microspectroscopy was used to characterize amorphous silica plastic behavior. Using a correlation between Raman spectrum and density, a map of the local residual indentation-induced densification is obtained. The existence of a densification-induced hardening is also evidenced through a diamond anvil cell experiment. Such observations are not accounted for by the previously proposed hardening-free pressure-dependent yield criterion based on indentation curves. These results open the way to more accurate description of a constitutive law for amorphous silica.
\end{abstract}

*Electronic address: damien.vdb@saint-gobain.com 


\section{INTRODUCTION}

Brittleness is a well-known and extensively studied characteristic of silicate glasses [1]. Comparatively, their plastic behavior has remained far less explored. Yet, the latter determines the contact mechanics of the glass surface [2] which is of primary interest for many micron-scale applications.

For obvious historical reasons, the standard vision of plasticity is still largely focused on crystalline materials (especially metals), where irreversible strain is triggered by shear stress and accommodated through volume-conserving dislocations motion [3]. Glasses do not fit into this frame for two different reasons. First, because of their amorphous nature there is no direct equivalent to these dislocation-based mechanisms [1]. Second, being amorphous, their structures, significantly more open than those of the chemically equivalent crystals 1], makes them prone to densify. Indeed, silicate glasses densify [4] while experiencing a plastic behavior that can be activated even by a purely hydrostatic loading [5]. This last characteristic leads to a distinction between normal and anomalous glasses, which exhibit respectively little and large densification, as well as different indentation fracture patterns 6, 7]. For fused silica (density 2.2 g. $\mathrm{cm}^{-3}$ ), the most anomalous silicate glass of industrial interest, this densification process is far from being negligible [8]. It is acknowledged to densify up to about $20 \%$ under mechanical stress [9]. Although this anomalous behavior of fused silica has been known for half a century [10], in-depth investigations were limited by the brittle nature of silica, which restricts the characteristic size of plastic deformation to micrometer scale [11]. Indeed, evidences of the densification process at room-temperature can only be obtained experimentally in confined geometries, such as in a Diamond-Anvil Cell 5] (DAC) or under an indenter [4, 10], which do not allow direct access to local stress/strain data through the commonly used macroscopic techniques.

We show here that, for amorphous silica, such local data of primary importance can be obtained experimentally through Raman microspectroscopy, which has recently proven particularly interesting as a method for local material characterization [12, 13]. Through the combination of a specific method to obtain unaltered cross-sections of indentations with Raman microspectroscopic mappings, we provide a map of the local indentation-induced densification in a fused silica sample. This experimental data on the local strain state of the material constitutes a relevant sieve for the validation of a constitutive law for amorphous 
silica $9,14,15,16,17,18,19]$.

The most sophisticated constitutive law for amorphous silica [15] does not account for these results. We suggest it be refined further by taking into account amorphous silica densification-induced hardening, which we also evidence here with a DAC experiment.

\section{EXPERIMENTAL PROCEDURE}

\section{A. Using Raman spectrum as a density gauge}

Between 200 and $750 \mathrm{~cm}^{-1}$, the Raman spectrum of silica glass consists in three bands (Fig. 11 contrasts the Raman spectrum of non-densified fused silica with the spectrum of largely indentation-densified silica glass). The main band at $440 \mathrm{~cm}^{-1}$ results from the symmetric stretching mode of bridging oxygens between two Si atoms. This band is intense, and affected by the densification process through a decrease of the inter-tetrahedral angles Si-O-Si [20, 21], but it was shown to be also very sensitive to elastic loading [22] (such as the residual elastic strain expected in indentation).

The defect lines $\mathrm{D}_{1}$ and $\mathrm{D}_{2}$, at 492 and $605 \mathrm{~cm}^{-1}$, are respectively attributed to the breathing modes of the four-membered and three-membered rings [23]. Their intensity ratio was previously used in literature as an indicator for "fictive temperature" [24, 25, 26], a slight change of which corresponds to a slight change in density. Moreover, they shift in position towards higher wavenumbers with increasing density, as the associated breathing modes get frustrated by the lack of free volume.

The $\mathrm{D}_{2}$ line is particularly useful because it has almost no overlap with the main band. Sugiura et al. 27] correlated the position of the $\mathrm{D}_{2}$ line with the ratio of the sample density $\rho$ to its initial density $\rho_{0}$. From their work, we get the following empirical relation:

$$
0.143 \log _{10}\left(\frac{\rho}{\rho_{0}}\right)=\log _{10}\left(\frac{\nu}{\nu_{0}}\right)
$$

$\nu$ and $\nu_{0}$ being respectively the final and the initial band position. The residual density evaluated through this relation accounts for both irreversible and elastic densification due to residual elastic strain. However, several works [22, 27] evidenced that the $\mathrm{D}_{2}$ line position is only marginally sensitive to residual elastic strains. 


\section{B. Diamond-Anvil Cell geometry}

An amorphous silica (Saint-Gobain Quartz IDD) splinter with a ten-micron characteristic length was used as experimental sample. It was introduced into a DAC apparatus along with a very small piece of ruby. A Raman spectrum of the sample was acquired prior to the fillingup of the cell compartment with the pressure transmitting fluid. We used a transmitting fluid close to 1:5 methanol-ethanol mix, which insures hydrostaticity up to 16 to $20 \mathrm{GPa}$ 28]. That choice was motivated by the fact that the densification process in amorphous silica is expected to saturate between 20 and $25 \mathrm{GPa}$ under hydrostatic loading [29].

The sample was then loaded to a maximal applied hydrostatic pressure evaluated to 14 GPa, using the shift of ruby fluorescence bands. After complete unloading, the Raman spectrum of the sample was measured.

\section{Indentation geometry}

Samples were prepared according to the following procedure. Plates of amorphous silica (IDD, Saint-Gobain Quartz), of dimension $10 \times 10 \times 0.4 \mathrm{~cm}^{3}$, were indented with an instrumented microindentation device allowing surface visualization. These plain 2-kg-Vickers indents allowed us to gain access to top views of the densified area.

2-kg-Vickers indents were also performed on a previously subcritically-grown crack, as described on figure 2. This provided us, on the crack surface, with a cross-section view of the densified material area located under the indent. In order to minimize the mechanical perturbation due to the presence of the crack, we performed a symmetrical loading. The indenter was thus positioned so that one of the Vickers pyramid diagonals be superimposed with the crack. Indentation curves obtained for on-crack indentations were similar (figure 3] shows that both indentation curves almost coincide) with that obtained for plain 2-kgVickers indentations, showing that the presence of a crack did not noticeably affect the indentation process.

We used a Raman microspectroscopy device (RM1000, Renishaw) including a $30 \mathrm{~mW}$ $514 \mathrm{~nm}$ Argon laser, under a $\times 100$ objective. The laser beam was focused on the surface

without confocal system. We however checked on one section view that the mapping obtained using confocal system agreed with that obtained without confocal system. We evaluated the 
excited volume of amorphous silica to approximately $2 \times 2 \times 5 \mu \mathrm{m}^{3}$. The characteristic size of that scanned volume, which directly depends on the laser wavelength, is too large to map an elastoplastic nanoindent. However, it can easily be used on microindents, such as that obtained with 2-kg-Vickers indents. Under the assumption that microcracks have no significant effect on the indentation-induced strain field, such experimental data can be used to validate an elastoplastic constitutive law for amorphous silica.

After checking densification symmetry with respect to the indenter geometry, we performed surface mappings. Owing to the symmetry of the Vickers indenter, we first mapped one eighth of the densified area on three different plain indents. We thus recorded spectra in 13 locations with a $10 \mu \mathrm{m}$-spacing, according to Fig. 4, during $1500 \mathrm{~s}$ each.

We then mapped half of the densified area on three different section views, recording spectra in 42 locations with a $5 \mu$ m-spacing, according to Fig. 5. We also performed similar measurements on subsurface positions in order to complete the section map. These measurements are to be compared with those obtained under indent edges on top view mappings. For each spectrum, Raman scattering signal was accumulated during $900 \mathrm{~s}$.

\section{RESULTS}

\section{A. Diamond-anvil cell geometry}

Figure 6] contrasts the initial spectrum of the sample (plain line) and that obtained after a 14 GPa hydrostatic loading (dotted bold line). Using relation (11) between the $\mathrm{D}_{2}$ line shift and densification, the densification experienced by the sample is derived directly from the spectra measured before and after hydrostatic loading. Thus the sample experienced a densification of approximately $10 \%$.

\section{B. Indentation geometry}

The $\mathrm{D}_{2}$ peak shift was then used as a density estimator according to equation (11). A typical top view map is displayed on Fig. 4. A typical cross-section map is displayed on Fig. 5.

The iso-densification lines obtained for the top view map (Fig. 4) are concentric. Their shape does account for the anisotropy of the Vickers pyramid. Indeed, densification is 
more pronounced under the indenter edge than it is under the indenter face. This result is consistent with the fact that shear stress (necessarily more intense under acuter parts of the Vickers pyramid) enhances densification [30].

The iso-densification lines obtained for the cross-section map (Fig. 5) are shaped into "concentric bowls", which agrees with the optical observations by Hagan and Van der Zwaag

6]. Quantitatively, we evaluated the densification just under the indenter tip at around $16 \%$. These results are consistent with the saturation of the densification process that is accepted to occur around $20 \%$ 9]. Moreover, we notice that the subsurface measurements are consistent with those obtained along the indent diagonal on top view maps. This validates that the indents performed on pre-existing cracks are indeed reasonably equivalent to plain indents. The densification just under the indenter tip was again estimated to approximately $16 \%$, in agreement with the previous measurement.

\section{DISCUSSION}

\section{A. Yield criterion for amorphous silica}

In most crystalline materials, plasticity occurs when the shear component of the applied stress reaches a yield value. Formally, this yield criterion can be written :

$$
f(\underline{\underline{\sigma}})=\tau-Y
$$

where $\tau$ represents the intensity of shear stress and $Y$ the yield strength. As long as $f(\underline{\underline{\sigma}})$ remains negative (i.e. as long as the shear stress remains smaller than the material yield strength), the material behaves elastically, while it may behave plastically only when $f(\underline{\underline{\sigma}})=0$. However, such a criterion cannot account for the silica glass densification under purely hydrostatic loading [5]. In order to do so, a dependance of $f(\underline{\underline{\sigma}})$ to the hydrostatic pressure $p$ has to be taken into account.

Recently, a hydrostatic-pressure-dependent yield criterion was proposed to describe the plastic behavior of dense amorphous materials like silica glass [9, 14, 15, 18, 19]. This plastic yield criterion, $f(\underline{\underline{\sigma}})$, can be written:

$$
f(\underline{\underline{\sigma}})=\alpha p+(1-\alpha) \tau-Y=-\frac{\alpha}{3} \operatorname{Tr}(\underline{\underline{\sigma}})+(1-\alpha) \sqrt{\frac{\underline{\underline{\underline{s}}: \underline{\underline{s}}}}{2}}-Y
$$

where $\operatorname{Tr}(\underline{\underline{\sigma}})=\sigma_{11}+\sigma_{22}+\sigma_{33}$ and $\underline{\underline{s}}=\underline{\underline{\sigma}}-\operatorname{Tr}(\underline{\underline{\sigma}}) \underline{\underline{I}}$ is the deviatoric part of $\underline{\underline{\sigma}}$. 
This pressure-dependent yield criterion is parameterized by two physical characteristics. One of them is the yield strength, $Y$. The other one, known as the densification factor 16] $\alpha$, balances the respective influence of applied hydrostatic pressure, $p$, and shear stress, $\tau$.For $\alpha=0$, we obtain the Van Mises criterion (Eq. (2) ), which describes the densificationfree plasticity of metals and, for $\alpha=1$, we describe a densification-only plastic behavior triggered exclusively by hydrostatic pressure, provided the plastic behavior is associated. Hence the denomination of $\alpha$ as the densification factor: the greater $\alpha$, the more anomalous the material.

Under the assumption of an associated perfect-plastic behavior, Xin and Lambropoulos

15] adjusted these two parameters by comparing experimental load/displacement indentation curve with finite element simulations. With the values $\alpha=0.6$ and $Y=5.43 \mathrm{GPa}$, they reproduced their experimental data: $\alpha=0.6$ accounts for a large contribution of densification to plastic strain (even allowing fused silica to exhibit a slight contraction of the cross section while being uniaxially compressed); and the hydrostatic loading yield pressure, obtained for $\tau=0$, is consistent with DAC experimental results [27].

\section{B. Amorphous silica densification-induced hardening}

We provide here a simple argument proving that amorphous silica hardens. Let us assume amorphous silica has a perfect plastic behavior (that is no hardening), at least until it reaches saturation density (See figure 17). When hydrostatically loaded, before saturation occurs, the applied stress on amorphous silica will never exceed the material yield strength, which is the only applied stress at which plastic flow occurs in a perfect plastic material. Thus, amorphous silica has necessarily reached saturation density before it can reach an applied pressure higher than its yield stress. Our DAC sample was loaded above 9 GPa, which is the yield pressure $p_{0}^{Y}$ provided by Xin and Lambropoulos' model. However, its densification is evaluated to a mere $10 \%$, which is lower than both literature claims (20\%) and our measurements under the indenter tip (16\%) for the saturation density. This leads to the conclusion that amorphous silica indeed exhibits hardening. Moreover, similar DAC cycling-loading experiments would provide us with the evolution of amorphous silica yield criterion with material density along the hydrostatic pressure axis. 


\section{Densification map: a relevant local characterization of indentation-induced strain field}

Indentation experiments result in largely inhomogeneous stress distributions. The applied force (resp. the penetration) is merely the integral of local applied pressure (resp. vertical displacement) and as such simply proportional to the mean applied pressure (resp. vertical displacement). The exclusive use of such integral data may prove insufficient to identify the parameters of a constitutive law trying to account for more local strain measurements. Our Raman microspectroscopic maps may constitute a severe validation for such laws and it may help to gain insight on the refinements to perform.

To test the influence of the densification factor on the general shape of the residual densified area, we performed FE calculations, using the software Abaqus, to model a cone indentation (of semi-apical angle of $70.3^{\circ}$ — making it the "axisymmetric equivalent" to a Vickers indenter). Consistently with Xin and Lambropoulos' approach [15], the material plastic behavior was assumed perfect and associated. The saturation, accounted for by preventing the material from densifying or flowing any longer, occurs when material permanent densification reaches $16 \%$. Calculations were performed for several values of $\alpha$ ranging between 0.2 and 0.6. The yield strength, $Y$, was chosen to match the experimental value in hydrostatic conditions $(Y / \alpha=9.05 \mathrm{GPa})$. Residual densification maps for $\alpha=0.2$ and $\alpha=0.6$ are presented in Fig. 8. When comparing these two maps, we notice that the indentation-densified areas are markedly different. In the case $\alpha=0.6$, with a large dependence on hydrostatic contribution, the numerical results predict a small but almost entirely saturated densified area . However, in the less anomalous case $\alpha=0.2$, the densification area is larger but with a smoother transition towards the non-densified material. This contrast highlights the direct relevance of the density map for the identification of the constitutive law.

\section{The role of strain-hardening in amorphous silica plasticity}

Let us now compare our experimental density map with the numerical results proposed by the literature. The densified area obtained by Xin and Lambropoulos [15] with $\alpha=0.6$ and $Y=5.43 \mathrm{GPa}$ is qualitatively close to that provided by figure 8 (b). The general shape and 
extension of the densified area is recovered. This confirms that the densification contribution to the silica plastic behavior is significant [9, 14, 15]. However, the mainly saturated core with a sharp near-boundary density gradient largely contrasts with the more progressive densification of the experimental result. This shows clearly that the constitutive law for silica densification identified using indentation curves needs to be further refined to account for the indentation-induced densification map.

At least one of the assumptions made in the constitutive law has to be relaxed. We expect that taking into account the densification-hardening process, the existence of which we have demonstrated by DAC measurements, will result in the concentric "bowl-shaped" areas associated to the experimental smoother density gradient, without much change in the global dimensions of the densified area.

\section{CONCLUSION}

Raman microspectroscopy provides experimentally relevant local data on the indentationinduced densification in amorphous silica. They constitute a discriminant sieve for the identification of its constitutive law, as they cannot be accounted for by existing models identified through indentation curves. Our results also clearly evidence a densificationinduced hardening process. Taking it into account would allow one to model more accurately the plastic behavior of amorphous silica.

We are currently working on finite-element simulation to provide such a plastic criterion for amorphous silica behavior. On-going experimental work also investigates the use of microspectroscopic methods to provide useful information on the mechanical behavior of other more complex silicate glasses.

\section{Acknowledgments}

The authors would like to thank G. Duisit, R. Gy, S. Pelletier, G. Quérel and S. Roux for their help, advice and support.

[1] J. Zarzycki, Glasses and the vitreous state; Cambridge University Press, 1991. 
[2] A. Griffith, Philos. Trans. Roy. Soc., A221 163 (1920).

[3] A.H. Cotrell, Dislocations and Plastic Flow in Crystals; Clarendon Press, Oxford, 1953.

[4] F.M. Ernsberger, "Role of densification in deformation of glasses under point loading", $J$. Am. Ceram. Soc., 51 [10] 545-547 (1968).

[5] H. Sugiura and T. Yamadaya, "Raman scattering in silica glass in the permanent densification region", J. Non-Cryst. Solids, 144 151-158 (1992).

[6] J.T. Hagan and S. Van Der Zwaag, "Plastic processes in a range of soda-lime-silica glasses", J. Non-Cryst. Solids, 64 249-268 (1984).

[7] J.T. Hagan, "Cone cracks around vickers indentations in fused silica glass", J. Mater. Sci., 14 462-466 (1979).

[8] C.R. Kurkjian and G.W. Kammlott, "Indentation behavior of soda-lime silica glass, fused silica, and single-crystal quartz at liquid nitrogen temperature", J. Am. Ceram. Soc., 78 [3] 737-744, (1995).

[9] J.C. Lambropoulos, S. Xu, and T. Fang, "Constitutive law for the densification of fused silica, with applications in polishing and microgrinding", J. Am. Ceram. Soc., 79 [6] 1441-1452 (1996).

[10] E.W. Taylor, "Plastic deformation of optical glasses", Nature, 163323 (1949).

[11] Y.W. Rhee, H.W. Kim, Y. Deng, and B.R. Lawn, "Brittle fracture versus quasi plasticity in ceramics: A simple predictive index", J. Am. Ceram. Soc., 84 [3] 561-565 (2001).

[12] A. Kailer, K.G. Nickel and Y.G. Gogotsi, "Raman microspectroscopy of nanocrystalline and amorphous phases in hardness indentations", J. Raman Spectrosc., 30 939-946 (1999).

[13] S. DiFonzo, W. Jark, S. Lagomarsino, C. Giannini, L. DeCaro, A. Cedolla and M. Muller, "Non-destructive determination of local strain with 100-nm spatial resolution", Nature, 403 638-640 (2000).

[14] A. Shorey, K. Xin, K.H. Chen and J.C. Lambropoulos, "Deformation of fused silica : Nanoindentation and densification", Proc SPIE, 3424 72-81 (1998).

[15] K. Xin and J.C. Lambropoulos, "Densification of fused silica : Effects on nanoindentation", Proc. SPIE, 4102 112-121 (2000).

[16] M. Imaoka and I. Yasui, "Finite element analysis of indentation on glass", J. Non-Cryst. Solids, 22 315-329 (1976).

[17] I. Yasui and M. Imaoka, "Finite element analysis of indentation on glass (ii)", J. Non-Cryst. 
Solids, 50 219-232 (1982).

[18] F. Dahmani, J.C. Lambropoulos, A.W. Schmid, S.J. Burns and C. Pratt, "Nanoindentation technique for measuring residual stress field around laser-induced crack in fused silica", $J$. Mater. Sci., 33 4677-4685 (1998).

[19] K. Xin and J.C. Lambropoulos, "Spherical cavity expansion in densifying material", J. Appl. Phys., 94 6437-6441 (2003).

[20] M. Tomozawa, Y.K. Lee and Y.L. Peng, "Effect of uniaxial stresses on silica glass structure investigated by ir spectroscopy", J. Non-Cryst. Solids, 242 [2-3] 104-109 (1998).

[21] S. Mochizuki and N. Kawai, "Lattice vibrationnal spectra of vitreous silica densified by pressure", Solid State Communications, 11 [6] 763-765 (1972).

[22] T.A. Michalske, D. Tallant and W.L. Smith, "Raman study of silica glass under tensile stress", Phys. Chem. Glasses, 29[4] 150-153 (1988).

[23] F.L. Galeneer, "Planar rings in glasses", Solid State Communications, 44[7] 1037-1040 (1982).

[24] B. Champagnon, C. Chemarin, E. Duval and R. Le Parc, "Glass structure and light scattering", J. Non-Cryst. Solids, 274 81-86 (2000).

[25] R. Le Parc, B. Champagnon, Ph. Guenot and S. Dubois, "Thermal annealing and density fluctuations in silica glass", J. Non-Cryst. Solids, 293-295 367-369 (2001).

[26] B. Champagnon, R. Le Parc and Ph. Guenot, "Relaxation of silica above and below $\mathrm{T}_{g}$ : light scattering studies", Phil. Mag. B, 82 [2] 251-255 (2002).

[27] H. Sugiura, R. Ikeda, K. Kondo and T. Yamadaya, "Densified silica after shock compression", J. Appl. Phys., 81 [4] 1651-1655 (1997).

[28] J.M. Besson and P.P. Pinceaux, "Uniform stress conditions in the diamond anvil cell at 200 kilobars", Rev. Sci. Instrum., 50 [5] 541-543 (1979).

[29] A. Polian and M. Grimsditch, "Room-temperature densification of a- $\mathrm{SiO}_{2}$ versus pressure", Phys. Rev. B, 41 [9] 6086-6087 (1990).

[30] J.D. Mackenzie, "High-pressure effects on oxide glasses", J. Am. Ceram. Soc., 46 [10] 461-476 (1963). 


\section{Figure Captions}

Figure 1: Raman spectra for amorphous silica. The plain line spectrum was obtained with 2.2 g.cm ${ }^{-3}$ amorphous silica, while the dotted line corresponds to indentation-densified amorphous silica.

Figure 2: Experimental procedure allowing the obtention of a section view of the indented area. Using a scratch as surface defect (a), a subcritical crack is grown in the sample, using a double-bending loading geometry (b). Symmetrical indentation is then performed on the crack (c). Crack propagation is then completed under double-bending (d). Two twin sections of the indented area are then obtained.

Figure 3: Indentation curves obtained for both on-crack indentation and plain indentation. They almost superimpose which shows us that the presence of a crack did not significantly affect the indentation process.

Figure 4: Densification map of one eighth of a top view of an indentation-densified area obtained using the $\mathrm{D}_{2}$ band position indicator. The center of the indent is at coordinates $(0 ; 0)$. The bottom thin line figures the edge of the indent while the right thin line corresponds to the middle of the pyramid face. The crosses correspond to the measurement points. The bold lines are the iso-densification lines. Numerical values are given in percent. Iso-densification lines appear to be concentric and account for the indenter anisotropy, as densification appears more important under the indenter edge.

Figure 5: Densification map of half a section view of an indentation-densified area obtained using the $\mathrm{D}_{2}$ band position indicator. The center of the indent is at coordinates $(0 ; 0)$. The top bold line represents the sample surface. The crosses correspond to the measurement points. The black lines are the iso-densification lines. Numerical values are given in percent. Iso-densification lines appear to be shaped into concentric bowls in agreement with observations by Hagan et al. [6]

Figure 6: Raman spectrum obtained with an amorphous silica sample before (plain line) and after (dotted line) a 14 GPa hydrostatic loading.

Figure 7:Expected behavior of a perfect plastic material under hydrostatic loading. Before pressure reaches the yield pressure $\left(p_{0}^{Y}=9 \mathrm{GPa}\right)$ the sample does not densify. As its

plastic behavior is hardening-free, applied pressure cannot reach higher values than $p_{0}^{Y}$ until irreversible densification, $\Delta V / V$, reaches its saturation value $\Delta V /\left.V\right|_{\text {sat }}$. 
Figure 8: Residual densification maps obtained through FE simulation of a 2Daxisymmetric cone indentation. They were obtained for two different values of the densification factor, (a) $\alpha=0.2$ and (b) $\alpha=0.6$. Densification values are given in percent. The results by Xin and Lambropoulos [15] are similar to (b) 


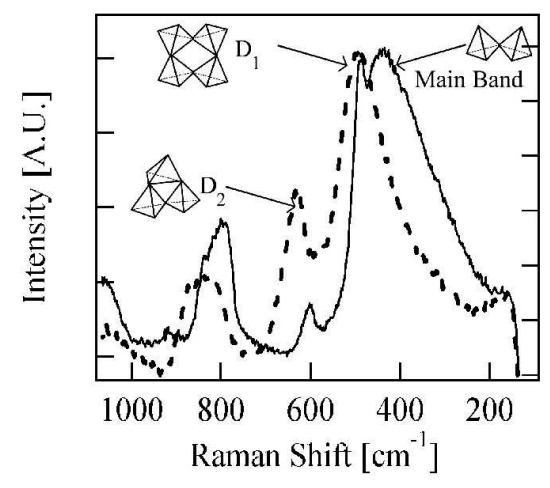

FIG. 1: Raman spectra for amorphous silica. The plain line spectrum was obtained with $2.2 \mathrm{~g} \cdot \mathrm{cm}^{-3}$ amorphous silica, while the dotted line corresponds to indentation-densified amorphous silica.
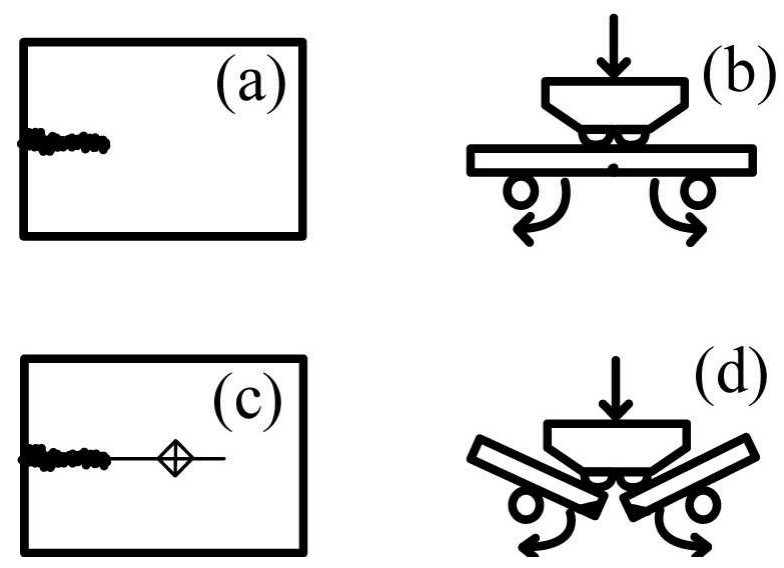

FIG. 2: Experimental procedure allowing the obtention of a section view of the indented area. Using a scratch as surface defect (a), a subcritical crack is grown in the sample, using a doublebending loading geometry (b). Symmetrical indentation is then performed on the crack (c). Crack propagation is then completed under double-bending (d). Two twin sections of the indented area are then obtained. 


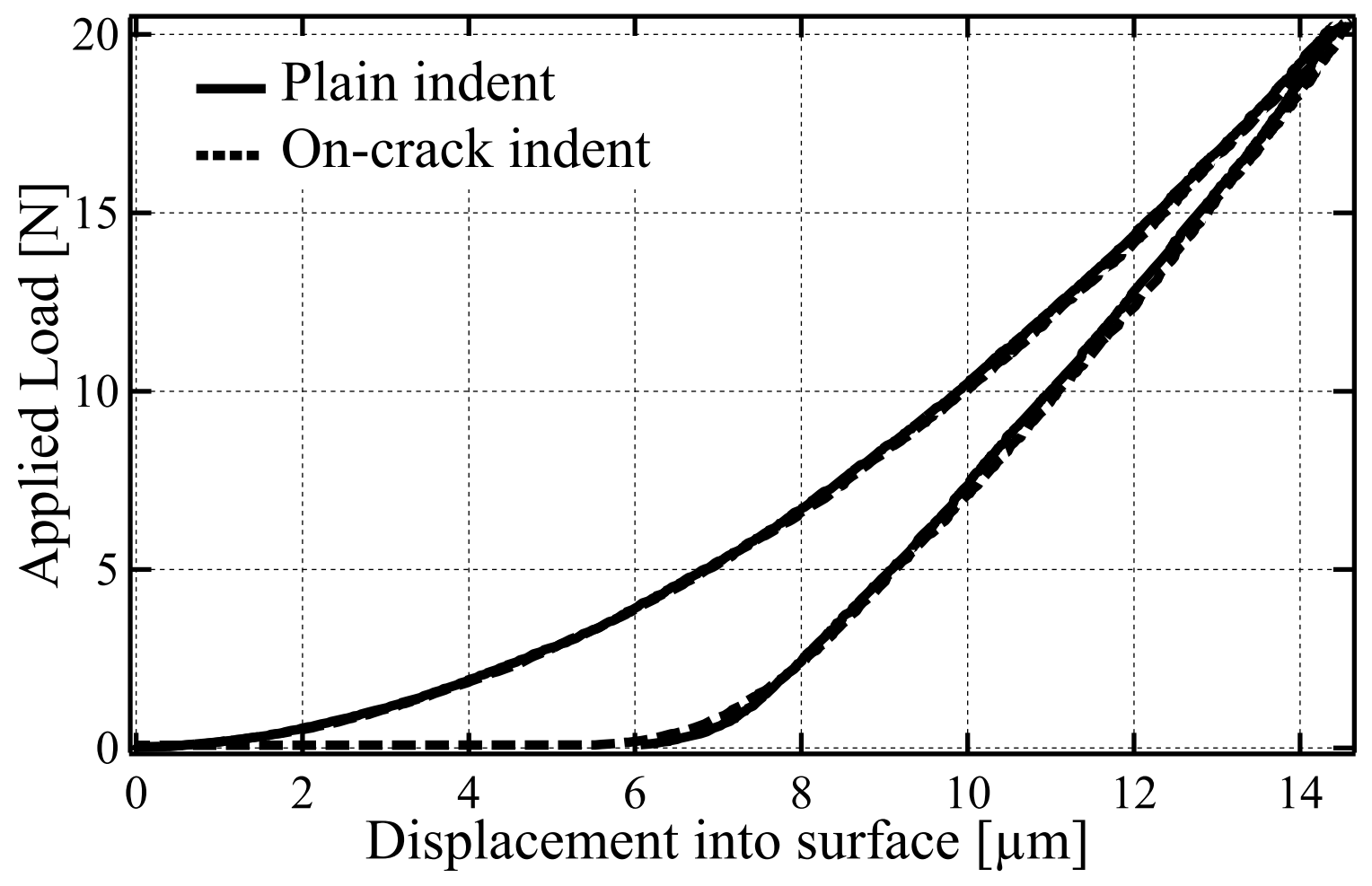

FIG. 3: Indentation curves obtained for both on-crack indentation and plain indentation. They almost superimpose which shows us that the presence of a crack did not significantly affect the indentation process. 

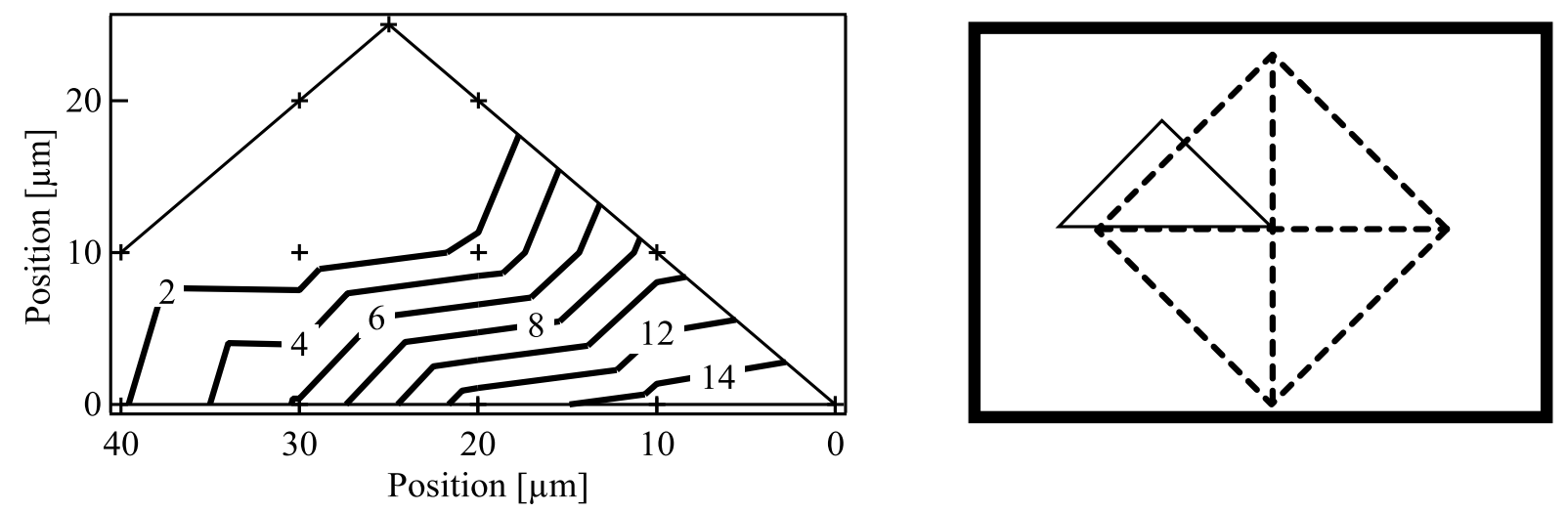

FIG. 4: Densification map of one eighth of a top view of an indentation-densified area obtained using the $\mathrm{D}_{2}$ band position indicator. The center of the indent is at coordinates $(0 ; 0)$. The bottom thin line figures the edge of the indent while the right thin line corresponds to the middle of the pyramid face. The crosses correspond to the measurement points. The bold lines are the isodensification lines. Numerical values are given in percent. Iso-densification lines appear to be concentric and account for the indenter anisotropy, as densification appears more important under the indenter edge. 


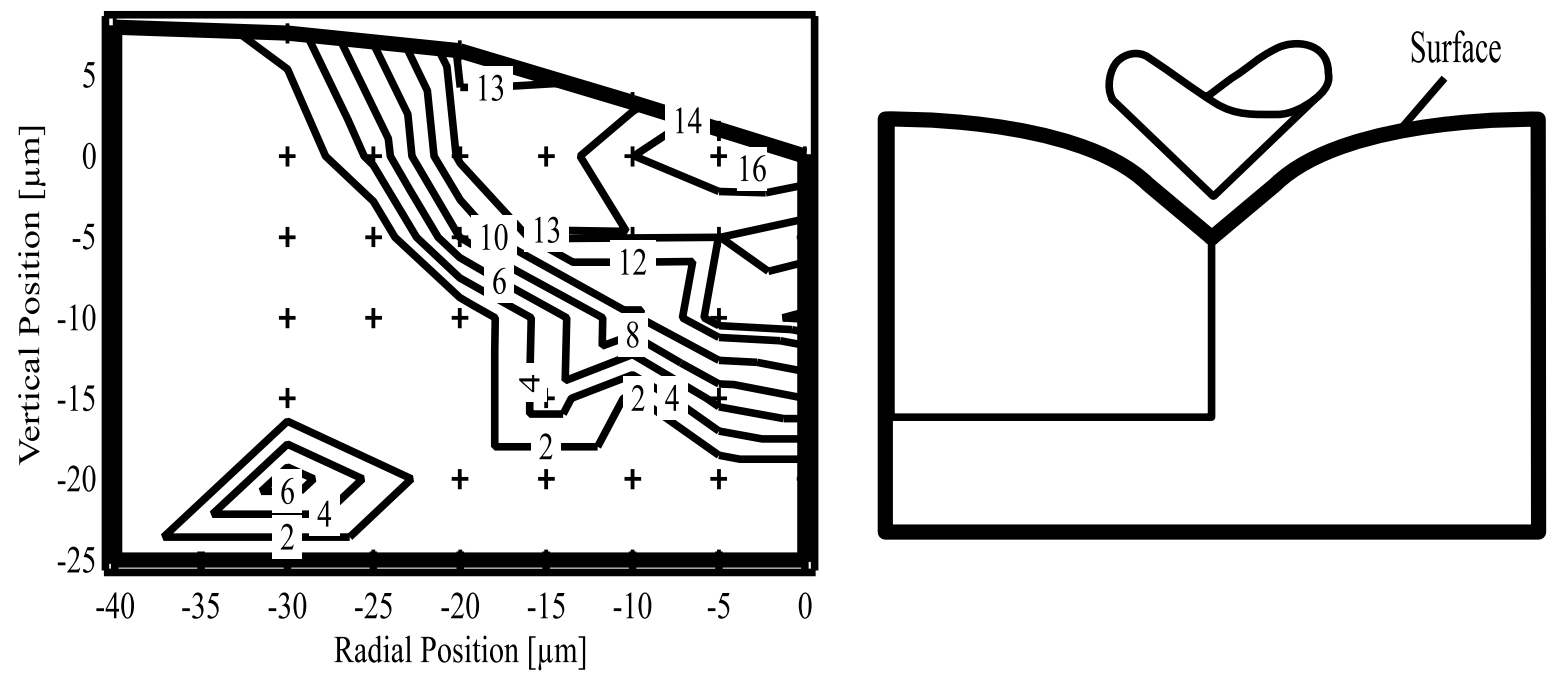

FIG. 5: Densification map of half a section view of an indentation-densified area obtained using the $\mathrm{D}_{2}$ band position indicator. The center of the indent is at coordinates $(0 ; 0)$. The top bold line represents the sample surface. The crosses correspond to the measurement points. The black lines are the iso-densification lines. Numerical values are given in percent. Iso-densification lines appear to be shaped into concentric bowls in agreement with observations by Hagan et al. [6]

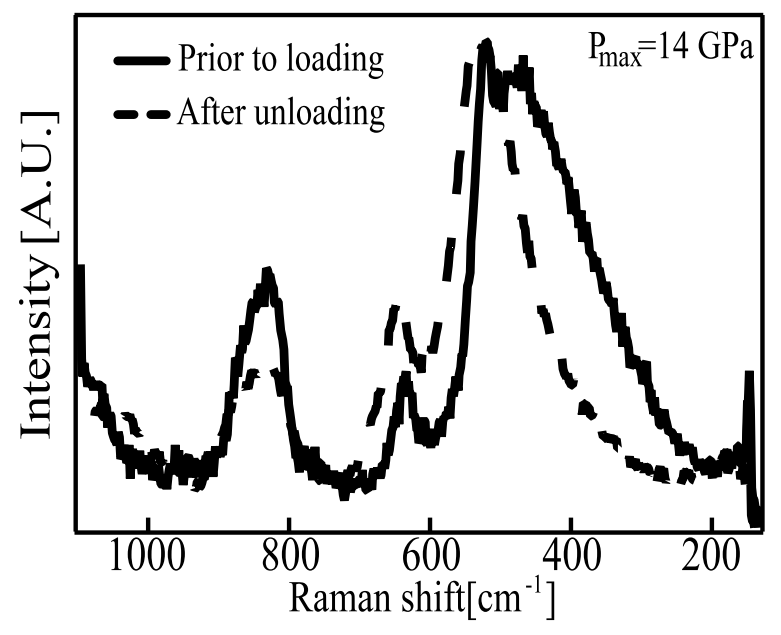

FIG. 6: Raman spectrum obtained with an amorphous silica sample before (plain line) and after (dotted line) a 14 GPa hydrostatic loading. 


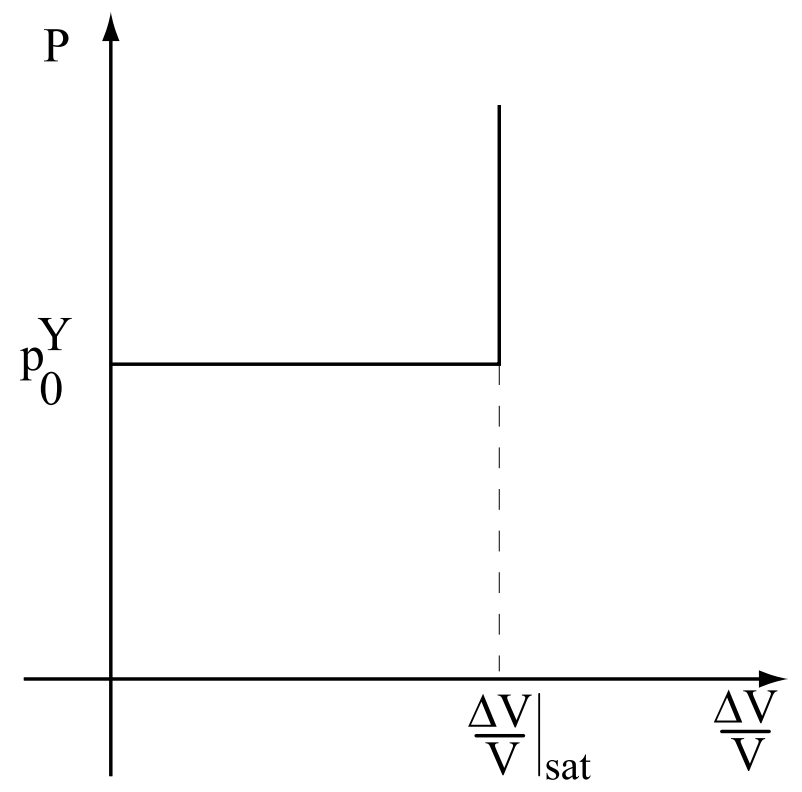

FIG. 7: Expected behavior of a perfect plastic material under hydrostatic loading. Before pressure reaches the yield pressure $\left(p_{0}^{Y}=9 \mathrm{GPa}\right)$ the sample does not densify. As its plastic behavior is hardening-free, applied pressure cannot reach higher values than $p_{0}^{Y}$ until irreversible densification, $\Delta V / V$, reaches its saturation value $\Delta V /\left.V\right|_{\text {sat }}$.
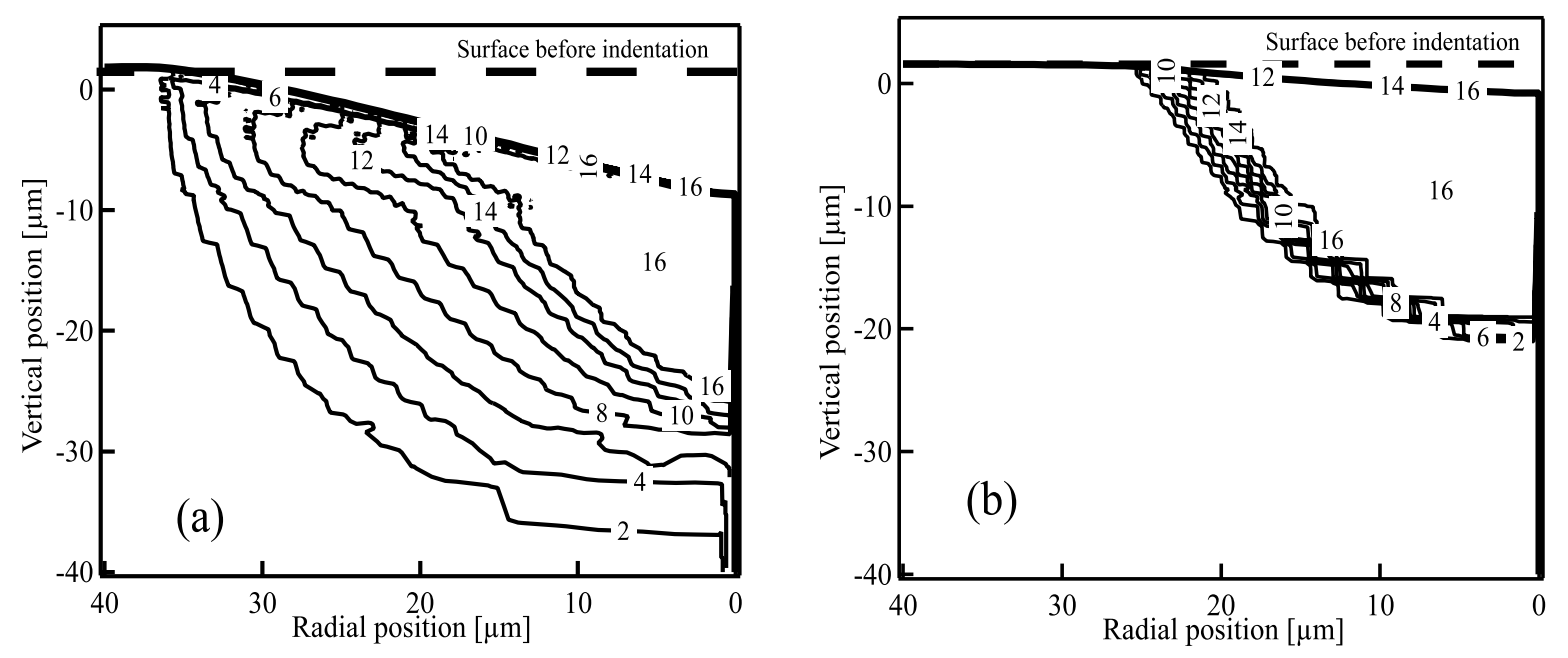

FIG. 8: Residual densification maps obtained through FE simulation of a 2D-axisymmetric cone indentation. They were obtained for two different values of the densification factor, (a) $\alpha=0.2$ and (b) $\alpha=0.6$. Densification values are given in percent. The results by Xin and Lambropoulos 15] are similar to (b) 